A N N A L E S Annales de Bretagne et des Pays de l'Ouest

\title{
Aux origines de la guerre de Succession de Bretagne. Documents (1341-1342)
}

Laurence Moal

\section{OpenEdition}

9 Journals

Édition électronique

URL : https://journals.openedition.org/abpo/5678

DOI : $10.4000 / a b p o .5678$

ISBN : 978-2-7535-8091-6

ISSN : 2108-6443

Éditeur

Presses universitaires de Rennes

Édition imprimée

Date de publication : 30 juin 2020

Pagination : 189-192

ISBN : 978-2-7535-8090-9

ISSN : 0399-0826

\section{Référence électronique}

Laurence Moal, «Aux origines de la guerre de Succession de Bretagne. Documents (1347-1342) », Annales de Bretagne et des Pays de l'Ouest [En ligne], 127-2 | 2020, mis en ligne le 30 juin 2020, consulté le 05 janvier 2023. URL : http://journals.openedition.org/abpo/5678; DOl : https://doi.org/10.4000/abpo. 5678 
teté " soumis par l'institution ecclésiale à un processus de contrôle et de normalisation qui lui assure, malgré un incontestable renouvellement, une pérennité dont la Charité d'Amiens constitue sans doute la plus belle expression; mais le saint moine dont Anschaire, aux dires de son propre biographe, s'efforce au Ix ${ }^{\mathrm{e}}$ siècle d'imiter le comportement (Frédéric Gross, p. 259-268), ou bien le saint prêtre emblématique de "l'idéologie sacerdotale " qui se développe en France au XVII ${ }^{\mathrm{e}}$ siècle (Milan Wenhert, p. 159-178), ou encore, au XVIII ${ }^{\mathrm{e}}$ siècle, le personnage hypostasié par le chapitre de Saint-Martin de Tours en vue de servir de défense et d'illustration à la collégiale (Christophe Maillard, p. 179-190), sont autant d'" avatars de Martin ", pour reprendre la formule de Bruno Judic, ou, plus largement, en écho au titre de l'ouvrage, autant de " nouveaux " Martin, qui viennent compléter la belle et déjà longue galerie de portraits martiniens offerts au public.

André-Yves BouRGES

GrAHAM-Goering, Erika, Jones, Michael, YeuRc'H, Bertrand, avec la collaboration de CHARON, Philippe (dir.), préface d'Yves CoATIVY, Aux origines de la guerre de Succession de Bretagne. Documents (1341-1342), coll. Sources médiévales de l'histoire de Bretagne, Presses universitaires de Rennes, Société d'histoire et d'archéologie de Bretagne, 2019, 344 p., ISBN : 978-2-7535-7789-3.

La collection des Sources médiévales de l'histoire de Bretagne avait jusqu'à présent permis d'enrichir la connaissance de l'histoire de la Bretagne médiévale avec plusieurs publications. Michael Jones et Philippe Charon étaient déjà à pied d'œuvre en 2014 pour l'édition dans la même collection de la thèse d'Hubert Guillotel consacrée aux Actes des ducs de Bretagne (944-1148), ainsi qu'en 2017 pour les Comptes du duché de Bretagne (1262-1352). Ce $9^{\mathrm{e}}$ ouvrage de cette précieuse collection est consacré, cette fois, aux origines de la guerre de Succession de Bretagne, une longue guerre civile de 23 ans (1341-1364), née d'une crise dynastique. Le duc Jean III meurt le 30 avril 1341 sans héritier direct et sans avoir désigné de successeur. Deux prétendants revendiquent alors le trône ducal : d'un côté sa nièce, Jeanne de Penthièvre, mariée avec Charles de Blois, neveu du roi de France; de l'autre son demi-frère, Jean, comte de Montfort.

Les trois éditeurs, Erika Graham-Goering, Michael Jones, Bertrand Yeurc'h, avec la collaboration de Philippe Charon, se sont attachés à rassembler, à transcrire et à éditer tous les principaux documents juridiques survivants sur le différend breton de la succession, proposant ainsi pour la première fois, sous une forme accessible, un éclairage sur le déclenchement du conflit entre 1341 et 1342. Les documents portent en effet sur une période charnière : l'affaire de la succession de Jean III et le dossier juridique de la querelle Blois-Montfort entre la mort du duc (30 avril 1341) et l'arrêt de Conflans (7 septembre 1341) ainsi que sur la marche à la guerre jusqu'en février 1342.

Une première version de certains des documents avait déjà été publiée par M. Jones il y a presque cinquante ans, mais dans une série de textes britanniques difficilement accessible. Depuis 2011, M. Jones s'emploie avec B. Yeurc'h à rééditer ces documents. Tous deux ont été rejoints en 2013 par E. Graham-Goering qui préparait sa thèse sur Jeanne de Penthièvre, ce qui lui a permis de trouver de nouveaux documents : un texte complet des arguments formels que Charles de Blois avait présentés au nom de sa femme pour succéder à Jean III, seulement connu à travers un résumé publié par Bertrand d'Argentré à la fin du xvl ${ }^{\mathrm{e}}$ siècle, ainsi qu'une copie, jusqu'ici inconnue, des témoignages donnés au nom de Jean de Montfort par des té- 
moins individuels. Cela permet de mettre en parallèle les arguments de Blois et ceux de Montfort. Qu'ils soient nouvellement découverts ou déjà connus, tous ces documents sont reconsidérés et apparaissent sous un nouveau jour en étant ainsi édités dans leur intégralité et dans le respect des préconisations de l’École des Chartes.

Le corpus de textes constitue la partie la plus importante de cet ouvrage (p. 73256). Chaque document est précisément présenté et annoté. L'ensemble est divisé en cinq grandes parties :

- Les arguments des deux candidats (I à IV, p. 73-156) : mémoires présentés aux noms de Jean de Montfort et Charles de Blois : cela permet de comparer les deux stratégies des deux prétendants.

- La protestation de la dame de Cassel, la sœur de Jean de Montfort (v, p. 159).

- L'enquête et les dépositions des 220 témoins (VI à vIII, 163-227), ainsi que les procès-verbaux de l'interrogatoire des témoins. Ces témoignages en langue vernaculaire concernent notamment les pratiques successorales. La parole de ces témoins permet d'appréhender leur place dans la hiérarchie sociale ainsi que leur répartition géographique et constitue une source précieuse pour étudier les partis, les réseaux, les groupes qui structurent la société.

- La décision de la cour : l'arrêt de Conflans (Ix, p. 231-232).

- La faillite de la diplomatie et la marche vers la guerre (x à XVIII, p. 237256) durant l'hiver 1341-1342.

Trois textes sont en latin : le résumé des arguments présentés par Jean de Montfort pour succéder à Jean III, son demi-frère aîné, comme duc de Bretagne (III), le détail des arguments présentés par Jean de Montfort à Philippe VI (IV) et l'arrêt de Conflans (IX). Il est dommage qu'aucune traduction n'en soit proposée.

L'ensemble est complété par des notices prosopographiques bretonnes qui retracent la carrière des témoins bretons et rassemblent le plus d'éléments possible sur leurs homonymes. S'y ajoutent deux index (d'une part un index des noms de lieux et de personnes et un index de matières d'autre part), un répertoire des sources et une bibliographie, des cartes, tableaux et des planches en couleur (lettres, manuscrits, généalogie, gisant de Jean III, sceau de Jeanne de Flandre...). Le livre se termine par une présentation des sources et de la bibliographie, dans laquelle on peut regretter l'absence d'Auray, 1364. Un combat pour la Bretagne, pourtant préfacé par M. Jones.

Ce recueil est précédé d'une importante introduction qui apporte un commentaire approfondi sur l'origine et la nature du différend mais aussi sur la chronologie des événements ainsi que sur le traitement historiographique de cette phase juridique. Les éditeurs rappellent les conditions dans lesquelles débute le procès, le 24 ou 27 août, sous les auspices de deux pairs ecclésiastiques, Hugues d'Arcy, évêque de Laon, et Pierre d'André, évêque de Noyon. L'ensemble de la procédure est terminé en deux semaines et le 7 septembre a lieu l'arrêt de Conflans. Les témoins déposent ensuite. Jean de Montfort quitte Paris à partir du 6 septembre, la veille du verdict. Ce n'est pas sa fuite qui a mis un terme à la procédure comme on l'a souvent cru. Le nombre de témoins est équivalent des deux côtés alors qu'on a considéré par erreur que Montfort n'avait pas eu de témoins individuels.

Comme le soulignent les éditeurs, la question n'est pas de savoir qui a raison mais d'appréhender la complexité des arguments et des contre-arguments. L'argument du camp montfortiste est fondé sur la coutume générale du royaume de France. Il met en valeur le statut de la Bretagne en tant que duché-pairie depuis 1297 et le principe de la souveraineté française en Bretagne. En même temps, le statut du duc est assimilé à celui du roi : la Bretagne était en effet jadis un royaume, argument déjà avancé en 1336 par Jean III contre la comtesse de Saint-Pol pour justifier ses droits comme duc et pair. L'argument du camp Blois-Penthièvre s'ap- 
puie quant à lui sur la coutume de Bretagne : les femmes peuvent accéder au trône contrairement à l'usage dans le royaume de France. Le statut de la Bretagne n'est pas déterminé de la même manière que les autres grandes seigneuries. Les deux partis utilisent en fait l'ambiguïté du statut ducal pour s'orienter vers des conclusions différentes (p. 25). Cette comparaison des arguments juridiques s'avère précieuse à plus d'un titre, notamment pour mieux comprendre le contexte social et politique. La comparaison est faite avec la succession de Limoges, une plus petite affaire d'héritage également contestée, la succession étant venue à Jean III par sa mère Marie de Limoges, première épouse d'Arthur II (p. 29).

Portant sur le statut des héritières, le débat concerne des problématiques très actuelles au croisement de l'histoire politique, de l'histoire des femmes et de l'histoire du genre. On se demande en effet si les femmes peuvent succéder au duché et si le fait que Jeanne soit une femme lui donne une préséance sur Jean de Montfort. Pour le camp Blois-Penthièvre, cela ne fait pas de doute. Il se base sur des précédents : Havoise en 1066, Berthe en 1148, Constance en 1166, Alix en 1203. Le camp Montfort lui objecte qu'il n'existe pas d'exemple depuis la pairie. Il ne nie pas la représentation mais prétend que c'est une coutume applicable aux sujets ducaux seulement, non au duc lui-même qui doit être jugé en suivant la coutume de Paris (p. 101, n. 342). En outre, s'il admet la représentation, " cela ne donnerait à Jeanne que les droits d'une sœur aînée de Jean de Montfort. Puisque la primogéniture bretonne donnait la préférence à la primogéniture masculine, elle arriverait toujours en deuxième place après Jean de Montfort en tant que fils survivant le plus âgé " (p. 26). Les montfortistes soutiennent qu'une femme ne peut gouverner les hommes à cause de "la fragilité de son corps ", elle ne peut assumer des responsabilités militaires : "Et est la duché parrie, en laquelle fame ne doit succeder [...]. Or est il certain que les fames, pour cause de leur condition et de l'inperfection de leur sexe, ne puent faire les choses dessus dictes " (p. 83). Les responsabilités d'un duc-pair ne pourraient être remplies par une duchesse-paire. Pour les Penthièvre en revanche, quand les femmes " ont juridiction de tenir héritaige, elles peuvent juger ». La question du mariage de l'héritière est également abordée. L'union d'un duc ou héritier à une noble étrangère ne pose pas de problème, contrairement au mariage d'une duchesse avec un étranger qui pourrait avoir une influence néfaste pour le duché. C'est la raison pour laquelle le promis doit être approuvé par les membres du cercle social (p. 27). Le mémoire de Jeanne de Penthièvre et Charles de Blois prétend qu'en prévision de leur mariage, contracté le 4 juin 1337, " furent requis tous les barons et les grands seigneurs du païs d'ulx y assanter pour ce que tous eulx du pays verent clairement qu'elle estoit et debvoit estre vroye heritiere de Bretaigne par la coustume du païs ". Cette ratification publique a continué le jour de leur mariage. Les évêques bretons ont également été consultés après la mort de Jean III et sept d'entre eux ont considéré que Jeanne était héritière légitime.

Les éditeurs traitent de manière très détaillée l'historiographie relative au procès de 1341. Pierre Le Baud est le premier à reconnaître que l'essentiel des revendications des Montforts est contenu dans le Songe du Vergier, version vernaculaire élargie du Somnium Viridarii, qui porte sur un débat scolastique entre un chevalier et un clerc sur la nature et l'exercice de l'autorité politique, constituée à la demande de Charles V vers 1370. Cette analyse permet d'affirmer que les arguments de Montfort ont été examinés et adaptés pour être utilisés dans le débat du clerc qui attaque la position du roi et du chevalier qui soutient la position du roi. Le différend sur la succession a été repris dans bon nombre de chroniques, en particulier celle de Froissart. Les manuscrits d'Amiens et de Rome montrent qu'il a obtenu des informations de première main sur les affaires bretonnes, des informateurs oraux comme Even Charruel en 1373-1374 et un des docteurs de Charles de Blois, Guillaume 
de Saint-Mesmin en 1388. Les généalogies constituent un élément central des tentatives des chroniqueurs pour expliquer la crise mais beaucoup de ces généalogies sont fausses. Le Bel et Froissart considèrent que Jean est le demi-frère maternel de Jean III et donc sans lien avec la lignée de Bretagne. Pour l'auteur de La Chronique des quatre premiers Valois, Jeanne de Penthièvre est placée dans une succession directe plutôt que collatérale. Ces erreurs ne sont pas forcément le résultat d'une entreprise partisane mais tendraient à prouver l'efficacité de la propagande des Valois.

L'étude des origines du conflit dans l'historiographie permet de réexaminer les initiatives politiques et militaires communément attribuées à Montfort. Le récit de Froissart de la chevauché de Jean de Montfort à la réception de la nouvelle de la mort de Jean III, repris ensuite par Le Baud et Bouchart, doit être remise en cause. Il existe peu de preuves d'un engagement militaire au cours de l'été 1341. Au contraire, l'inaction de Blois, vue par Froissart comme une stratégie, lui aurait été suggérée par son entourage. Mais en l'absence de source extérieure, les éditeurs invitent à la prudence. Ils comparent ensuite la séquence de six événements-clés de l'ouverture du conflit dans différentes chroniques : la chevauchée de Jean de Montfort, l'ouverture d'un examen juridique des revendications, le verdict royal, la fuite de Jean de Paris, le retournement montfortiste vers l'Angleterre, l'investiture de Charles et/ou de Jeanne par Philippe VI impliquant des hommages. Cette comparaison montre que les chroniques sont de " mauvais guides sur la causalité des premiers événements " mais " les auteurs étaient au moins profondément intéressés par cette causalité, de sorte qu'ils la retravaillaient continuellement pour tenter de mettre de l'ordre et de la logique à une série complexe d'événements " (p. 45-46).

Les différents points de vue sur la demande d'une aide militaire à l'Angleterre sont ensuite passés en revue. Dans les chroniques, la fuite de Jean de Paris a lieu à différents moments, avant ou après l'arrêt de Conflans. Selon Le Bel et Froissart, le départ se situe à la suite d'une entrevue avec Philippe VI. La fuite serait donc liée au verdict du procès, mais il s'agit d'un point de vue isolé. Le déroulement du processus, tel qu'il est désormais documenté, suggère que si Montfort est parti avant le verdict, c'est seulement à la toute fin plutôt que juste après l'annonce du procès, un point de vue adopté par la Chronique anonyme de sire Bertrand du Guesclin (p. 47).

Les événements de 1341-1342 et le chemin qui mène la Bretagne à la guerre sont enfin passés en revue : l'arrestation de Jean de Montfort, l'évolution des relations avec l'Angleterre après la mort de Jean III, le rôle de Jeanne de Flandre dans la prise de commandement de la cause montfortiste. L'attitude de Jeanne est apparemment conciliante comme l'indiquent les trêves successives et les négociations avec Philippe VI pour gagner du temps jusqu'à ce qu'Édouard III puisse livrer l'aide militaire qui conduit à l'occupation des villes, ports et châteaux de la côte bretonne par les Anglo-Bretons. Avec cette campagne militaire, la guerre civile est " devenue inextricablement absorbée par le cadre plus large de la guerre générale anglo-française " (p. 55). Cette très riche introduction se termine par les conventions d'édition.

L'ensemble de cet ouvrage constitue un travail d'érudition très solide qui va bien au-delà d'un simple instrument de travail. Il est à consulter en vue de recherches nouvelles notamment sur les témoignages qui ouvrent de très nombreuses perspectives : gouvernement ducal, pratiques sociales, généalogie, langue, rôle de la mémoire, utilisation des preuves historiques... Des pans entiers de l'histoire bretonne ressortent mieux éclairés. Mais l'intérêt dépasse largement les limites de la Bretagne en particulier avec des informations sur le comté de Montfort et la châtellenie de Neaufle ou sur l'histoire juridique. 